Classification

Physics Abstracts

$75.50 \mathrm{M}-67.65-64.70$

\title{
First-order transition in the instability of a magnetic fluid interface
}

\author{
J.-C. Bacri and D. Salin \\ Laboratoire d'Ultrasons (*), Université P. et M. Curie, \\ Tour 13, 4, place Jussieu, 75230 Paris Cedex 05, France
}

(Reçu le 2 mars 1984, accepté le 12 avril 1984)

\begin{abstract}
Résumé. - Nous avons observé l'instabilité de surface à l'interface entre un ferrofluide très concentré et un ferrofluide moins concentré. Expérimentalement, nous montrons que la transition entre l'interface plat et le réseau régulier de pics observé est une transition du premier ordre. Grâce à la forte valeur de la perméabilité relative $(\mu \sim 40)$ de notre ferrofluide nous observons un grand hystérésis à la transition.
\end{abstract}

\begin{abstract}
We have observed the surface instability of the interface between a highly concentrated ferrofluid and a weakly concentrated one. Our experimental data clearly show the first order character of the transition between a flat interface and the observed regular pattern of peaks. A large hysteresis effect is observed because of the high value of the relative permeability $(\mu \sim 40)$ of our concentrated ferrofluid.
\end{abstract}

\section{Introduction.}

Since the basic experiment of Cowley and Rosensweig [1], it is well known that, for a large enough vertical magnetic field, the initially flat and horizontal interface between a magnetic fluid and a second fluid becomes unstable and exhibits a stationary pattern of peaks. The crests of the peaks are arranged in a hexagonal array. Theoretical calculations [1-3] of the magnetic field threshold $H_{\mathrm{c}}$ and of the critical spatial wave number of the array are performed assuming a small periodic perturbation from the flat interface. These calculations are in reasonable agreement with the experiment, but the question of the height of the peaks at the threshold (and for larger field values) is still open. After much discussion [4-7], theoretical predictions conclude that, for a hexagonal array, a first-order transition occurs whatever the value of the relative magnetic permeability $\mu$ of the magnetic fluid : when the magnetic field $H$ is increased from zero, peaks are expected to appear with a finite height at $H=H_{\mathrm{c}}$; when $H$ is decreased from large values, peaks are expected to disappear at a lower field threshold $H^{*}$. The previous experiment does not confirm these predictions; this may be due to the smallness of the hysteresis effect $\left(\frac{H_{\mathrm{c}}-H^{*}}{H_{\mathrm{c}}} \ll 1\right)$. In fact hysteresis effects are expected to depend on $(\mu-1)^{2}$ (for $\mu$ of the

(*) Associated with the Centre National de la Recherche Scientifique. 
order of 1) and the usual ferrofluids do not have large enough initial magnetic permeabilities $(\mu<1.8$ in Ref. [1]). An ionic ferrofluid, manufactured by a chemical process [9], was used to prepare [10] a magnetic fluid phase with a large permeability $(\mu \sim 40)$. We report on the interface instability in such a magnetic fluid phase where we clearly observe a first-order transition with large hysteresis effects $\left(H_{c} / H^{*} \sim 1.35\right)$. We also develop a model to interpret our data.

\section{Theoretical survey.}

We consider the flat interface between two magnetic fluids submitted to a vertical magnetic field $H$. On this interface, small, thermally excited undulations take place; the magnetic flux is concentrated at the top of these undulations and the resulting forces tend to increase the perturbation still further. Interfacial tension and gravity tend to stabilize the interface. By considering small periodic undulations of the interface, the threshold of the instability of the flat interface can be expressed [1] in terms of a critical magnetization $M_{\mathrm{c}}$ and a critical wave number $k_{\mathrm{c}}$ :

$$
\begin{gathered}
\mu_{0} M_{\mathrm{c}}^{2}\left(1+\frac{1}{r}\right)=2 \sqrt{\Delta \rho g \sigma} \\
k_{\mathrm{c}}=\sqrt{\Delta \rho g / \sigma} .
\end{gathered}
$$

The international system of units is used, $\mu_{0}=4 \pi \times 10^{-7} \mathrm{SI}, g=9.81 \mathrm{~m} / \mathrm{s}^{2}, \sigma$ is the interfacial tension, $\Delta \rho$ is the specific mass difference between the two fluids and $r$ is a kind of relative permeability taking into account the non-linear dependence of the magnetization versus the magnetic field $H: r^{2}=\frac{B}{\mu_{0}^{2} H} \frac{\partial B}{\partial H} . B$ is the magnetic induction. In fact, the experimentally measured parameters are the nearest-neighbour distance of the peaks in the pattern which are related to the critical wavelength $\lambda_{\mathrm{c}}=2 \pi / k_{\mathrm{c}}$ and the critical magnetic field $H_{\mathrm{c}}, M_{\mathrm{c}}=\frac{\chi H_{\mathrm{c}}}{1+\chi D}$, where $D$ is the demagnetization factor of the sample and $\chi=\mu-1\left(B=\mu_{0} \mu H\right)$ the susceptibility of the magnetic fluid. Quantitative experimental evidence of the validity of equations (1) and (2) has been reported [1]; in such experiments, where the relative permeability $r$ never exceeded 1.8 , the authors did not mention any jump or hysteresis effects : the instability appears as a weak first-order transition (soft instability in the Russian literature [4-6]). The calculation of the amplitude of the periodic undulations, in the vicinity of the threshold, require further development of the equations and Shliomis [4] has shown, in the case of a one-dimensional wave only, that depending on the value of the relative permeability $\mu$ of the magnetic fluid, the transition can be either second order $(\mu<3.54)$ or first order $(\mu>3.54)$. Gailitis showed that this case is degenerate [5] and concluded [6] that a first-order transition occurs in the case of a hexagonal array of undulations (for any $\mu$ ) and in the case of a square array for large enough permeability $(\mu>1.09)$. He also predicted transitions between these two arrays. More recently [7], in a more rigourous mathematical treatment, Thomas and Thombly confirmed the Gailitis results but with a different value of $\mu$ for the separation between first- and second-order transition regimes in the case of the square array $(\mu=1.44$ instead of 1.09). In any event, while these theories predict the character of the transition (first or second order), they do not give either the width $\left(H_{\mathrm{c}}-H^{*}\right)$ of the hysteresis in the case of first-order transition, or the height of the peaks at the instability thresholds $\left(H_{\mathrm{c}}\right.$ and $\left.H^{*}\right)$. We will now describe our experiment and compare the results to these theoretical expectations. 


\section{Experiment.}

We use an aqueous solution of ferrofluid obtained by a chemical process $[8,9]$. Instead of the classical surfactant stabilization, this type of colloidal suspension of magnetite is stabilized by low polarizing cations. For certain concentration values in these countercations, our ferrofluid exhibits a phase separation between a rich concentration phase of magnetite grains (typical size $100 \AA$ ) and a low concentrated phase [10]. This concentrated phase, which is denser than the second phase $\left(\Delta \rho \simeq 500 \mathrm{~kg} / \mathrm{m}^{3}\right)$ settles at the bottom of our cell to a height of $d \simeq 200 \mu \mathrm{m}$. It is this concentrated phase which is our magnetic fluid, the overlaping fluid being only slightly magnetic. Our sample cell is $5 \mathrm{~cm}$ high, $1 \mathrm{~cm}$ wide and $L=200 \mu \mathrm{m}$ thick; the magnetic field $H$ being in the vertical direction, the demagnetization factor $D$ of the cell is $D=\frac{2}{\pi} \tan ^{-1}\left(\frac{L}{d}\right) \simeq 0.5$ [11]. From the deformation of a drop of concentrated phase surrounded by the low concentrated phase, in a magnetic field [10], we get the interfacial tension between the two phases $\sigma=2 \times$ $10^{-6} \mathrm{~J} \mathrm{~m}^{-2}$ and the ratio of their magnetic permeabilities $\mu_{2} / \mu_{1} \simeq 39$. As the low concentrated phase permeability is close to $\mu_{0}$, the susceptibility of the rich phase is $\chi=\mu-1 \simeq 38$. The observation of the interface between the two phases is performed with a microscope and a videosystem which has already been described elsewhere [10]. The magnetic field, produced by two magnetic coils, is increased step by step; for each field value we wait a few minutes. The measured height $p$ of one peak versus the applied magnetic field $H$ is given in figure 1 ; the circles correspond to increasing magnetic field, the crosses to decreasing magnetic field. These data clearly show the first order character of the transition and the large hysteresis effect $\left(H_{\mathrm{c}} / H^{*}=1.35\right)$. We

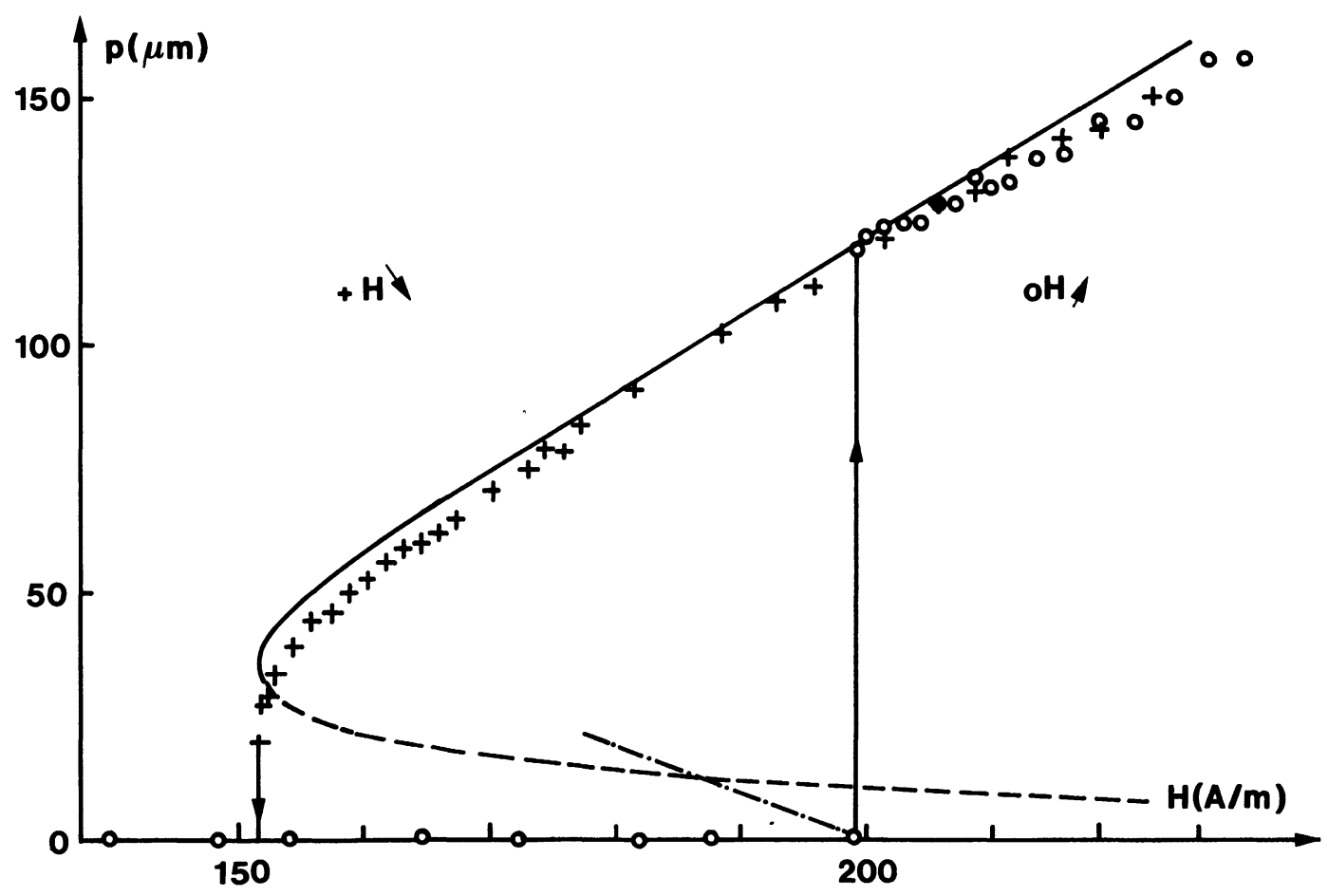

Fig. 1. - Height of the peak $p$ versus the applied magnetic field $H ;(0)$ increasing $H,(+) H$ decreasing. Lines are theoretical (see text). 
observe a single line of peaks (picture 1), with a spatial periodicity $\lambda_{\mathrm{m}}=160 \mu \mathrm{m}$ from one peak to its nearest neighbours; this value of $\lambda_{\mathrm{m}}$ does not change, to within $10 \mu \mathrm{m}$, over the whole range of field that we have covered. The fact that we observe only a single line of peaks is most likely due to the small thickness $(L=200 \mu \mathrm{m})$ of our sample : the wave vector $k$ is quantized in this direction as a multiple of $2 \pi / L$ and the critical spatial wavelength $\lambda_{c}$ of the peaks in our experiment is then $\lambda_{\mathrm{c}}^{-2}=L^{-2}+\lambda_{\mathrm{m}}^{-2}$, i.e., $\lambda_{\mathrm{c}}=125 \mu \mathrm{m}$. The agreement between our experimental results, at the threshold $\left(H_{\mathrm{c}}=198 \mathrm{~A} \mathrm{~m}^{-1}, \lambda_{\mathrm{c}}=125 \mu \mathrm{m}\right)$ and theory (expression (1) and (2)) is reasonably good : $H_{\mathrm{c}}=205 \mathrm{~A} \mathrm{~m}^{-1}, \lambda_{\mathrm{c}}=130 \mu \mathrm{m}$ with $\sigma, r=\mu, \chi, D, \Delta \rho$ given previously.

The first-order character of the transition that we observe is in agreement with theoretical expectations for the case of large $\mu[4,6,7]$, but we have neither a hexagonal nor a rectangular array, we have, in fact, the case of a two-dimensional array, with a dimension $(L)$ imposed by the boundaries. We have now to build a simple model to interpret the large hysteresis effect observed and the height $p(H)$ of the peaks versus the applied magnetic field. The resulting height of the peaks is due to a balance between magnetic energy and gravity and interfacial energies. We will try to evaluate the energy changes from a flat interface to an interface covered with peaks. As one can see in picture 1, the shape of the observed peaks is not that of small undulations of the surface, but appears with a conical form. Except for very small peaks $(p \leqslant 40 \mu \mathrm{m})$ the semiangle $\theta$ of each cone is nearly constant $(\operatorname{tg} \theta \sim 0.3)$. The energy change from a flat interface of basal area $L \times \lambda_{\mathrm{m}}$ to a peak of height $p$ on the same basal area can be written in a general form as

$$
E=2 A_{1} \sigma p^{2}+4 A_{\mathrm{G}} \Delta \rho g p^{4}-3 \mu_{0} A_{\mathrm{M}} M^{2} p^{3}
$$

where the 3 terms correspond respectively to interfacial, gravity and magnetic energy changes.

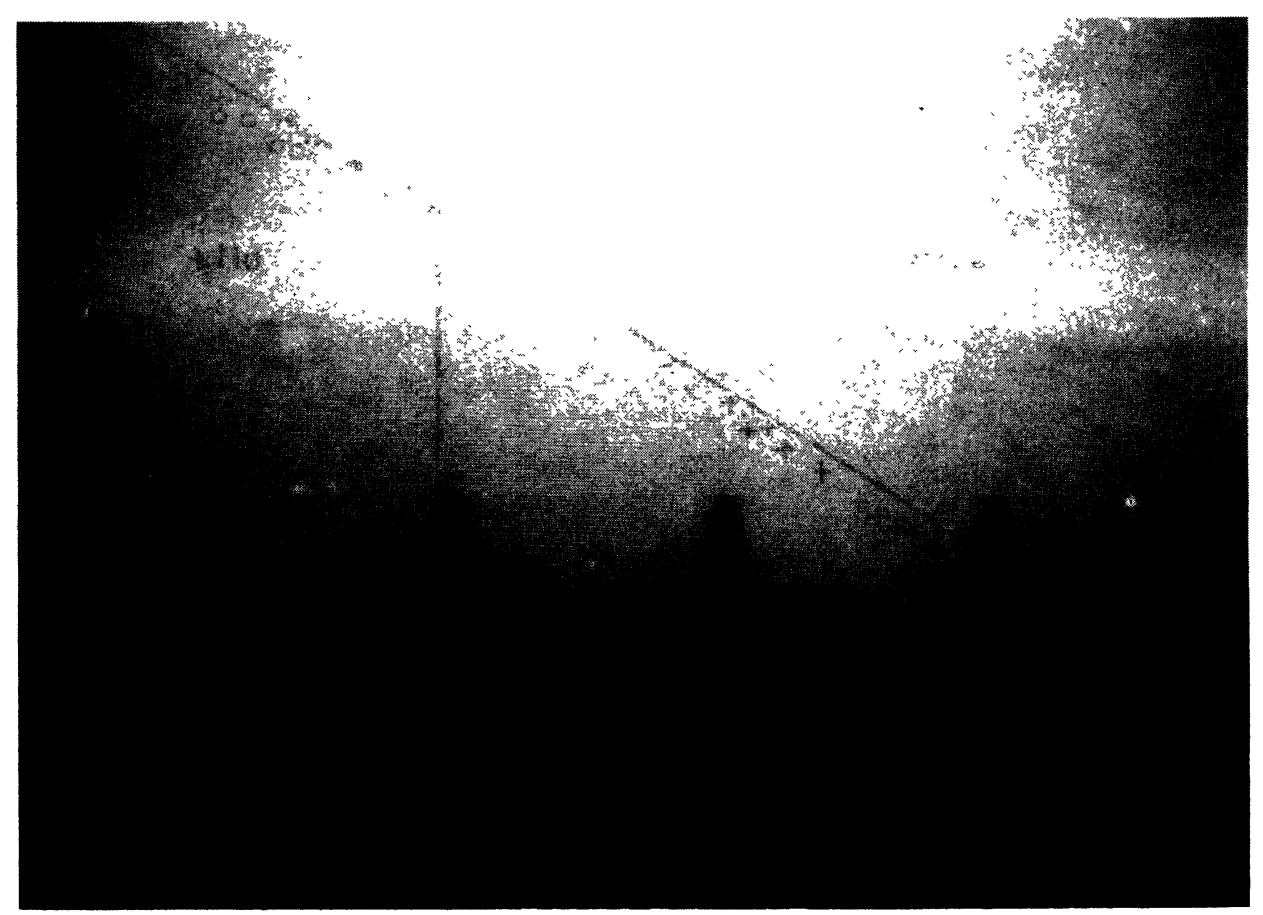

Picture 1. - Picture of the line of periodic peaks for a $220 \mathrm{~A} \mathrm{~m}^{-1}$ applied magnetic field. The mean distance between peaks is $160 \mu \mathrm{m}$. 
The peaks with different height $p$ are homothetic. $A_{\mathrm{I}}$ and $A_{\mathrm{G}}$ are geometrical factors depending only on $\theta$ (for example $A_{\mathrm{I}}=\frac{\pi \sin \theta}{2(1+\sin \theta)}, A_{\mathrm{G}} \simeq \frac{\pi}{36} \tan ^{2} \theta\left(1+\frac{\pi}{2} \tan ^{2} \theta \frac{p^{2}}{L \lambda_{\mathrm{m}}}\right)$ for a cone of revolution). For the magnetic term we assume that the peaks are independent (no interactions) and that $M$ is the magnetization corresponding to the applied magnetic field $H$; in fact $M$ is not constant throughout the peak and its surroundings, but we make the hypothesis that the magnetic energy is proportional to peak volume $\left(p^{3}\right)$ and $M=\frac{\chi H}{1+\chi D} ; A_{M}$ will be an adjustable parameter of our model.

For a given applied magnetic field $H$, the height $p$ of the peak is given by minimizing the energy change $E$ with respect to $p$. Extrema of $E$ are simply given as

$$
p=0 \text { and } p_{ \pm}=p^{*}\left(\left(\frac{H}{H^{*}}\right)^{2} \pm \sqrt{\left(\frac{H}{H^{*}}\right)^{4}-1}\right)
$$

where $p^{*}$ and $H^{*}$ can be expressed in terms of $\lambda_{\mathrm{c}}$ and $H_{\mathrm{c}}$ :

$$
p^{*}=\sqrt{\frac{A_{\mathrm{I}}}{A_{\mathrm{G}}}} \frac{\lambda_{\mathrm{c}}}{2 \pi} \text { and } H^{*}=\frac{\sqrt{A_{\mathrm{I}} A_{\mathrm{G}}}}{A_{\mathrm{M}}} H_{\mathrm{c}}^{2}
$$

The solution $p_{-}$corresponds to a maximum of energy and is thus always unstable $; p_{+}$and $p=0$ correspond to minima of energy. For $H<H^{*}, p=0$ is stable ( $p_{+}$does not exist). For $H^{* 2}<$ $H^{2}<\frac{3}{2 \sqrt{2}} H^{* 2}, p_{+}$is metastable and $p=0$ is still stable. For $H^{2}>\frac{3 H^{* 2}}{2 \sqrt{2}}, p=0$ is metastable and the solution $p_{+}$is the stable height. A complete understanding of the hysteresis effect in our first-order transition needs a connection, between our model and the previous theory $[1,3]$ in which $H_{\mathrm{c}}$ is the threshold of instability of small undulations. Since in our experiment $H^{*}$ is smaller than $H_{\mathrm{c}}$ (and also $H^{* 2}<3 H_{\mathrm{c}}^{2} / 2 \sqrt{2}$ ) the stable state for $H<H_{\mathrm{c}}$ ( $H$ increasing) must be $p=0$. We can now describe the behaviour of the interface for a cycle in magnetic field : increasing $H$ from zero, the flat interface $(p=0)$ persists until it becomes unstable $\left(H=H_{\mathrm{c}}\right)$; at this field value peaks appear with a stable height value $p_{+}\left(H_{\mathrm{c}}\right)$ (expression (4)). Increasing $H$ again, $p_{+}(H)$ increases. On decreasing $H$ from these high values, $p_{+}(H)$ decreases until it becomes unstable $\left(H=H^{*}\right)$ at a finite height value $p^{*}$; at this field value the height of the peaks falls off to zero and the flat interface persists for lower fields. Quantitatively, in our experiment $\tan \theta \sim 0.3$ except in the close vicinity of $H^{*}\left(H-H^{*} \sim 10^{-2} H^{*}\right)$ where $\theta$ increases up to a value $\tan \theta=1$ for the last field value where we still observe peaks, i.e. for $H=H^{*}$. The solid curve through the data corresponds to our model height $p_{+}(H)$ (expression (4)) with $p^{*}=43 \mu \mathrm{m}$. This value of $p^{*}$ is smaller than that computed with expression (5) $(66 \mu \mathrm{m}$ for $\tan \theta \sim 0.3)$ for a conical shape; this would mean that our geometrical factor $\sqrt{A_{\mathrm{l}} / A_{\mathrm{G}}}$ is not correct but that the field dependence is reasonably good except in the close vicinity of $H^{*}$ where $\theta$ increases; if this $\theta$ variation is taken into account, we get $p^{*}=18 \mu \mathrm{m}$ at the threshold $H^{*}$, in better agreement with our experimental value $(16 \mu \mathrm{m})$. The dashed dotted straight line corresponds to the theoretical development in the vicinity of $H_{\mathrm{c}}$ for small undulations [6,7]. This line is somewhat close to

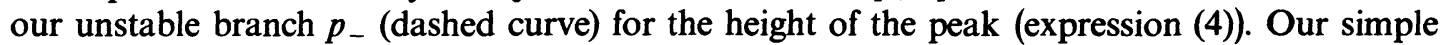
model is in reasonable agreement with our experimental data. To obtain better agreement a much more complicated computation of the magnetic energy for peaks of arbitrary shape and height would be required. 


\section{Conclusion.}

We have observed, for the first time, a first-order transition in the instability of a flat interface between two magnetic fluids. The large hysteresis effect is due to the high relative permeability of the magnetic fluid. We have developed a simple model in order to interpret the hysteresis effects and the height of the peaks. Agreement with our experimental data is reasonably good to within some not well defined geometrical factors.

\section{References}

[1] Cowley, M. D. and Rosensweig, R. E., J. Fluid Mech. 30 (1967) 671.

[2] Frenkel, J. I., Phys. Z. Sowjetunion 8 (1935) 675.

[3] Zelazo, R. E. and MelCheR, J. R., J. Fluid Mech. 39 (1969) 1.

[4] Zaitsev, V. M. and Shliomis, M. I., Proc. Acad. Sci. USSR 188 (1969) 1261.

[5] Gallitis, A., Magnit. Gidrodin. 5 (1969) 68.

[6] Gailitis, A., J. Fluid Mech. 82 (1977) 401.

[7] Twombly, E. and Thomas, J. W., IEEE Trans. Magn. 16 (1980) 214.

[8] Union Chimique Européenne, 6 rue Gutenberg, Bondoufle, 91032 Evry Cedex, France.

[9] MASSART, R., IEEE Trans. Magn. 17 (1981) 1241.

[10] BaCri, J.-C. and Salin, D., J. Physique Lett. 43 (1982) L-649.

[11] Rosensweig, R. E., J. Magn. Magn. Mater. 39 (1983) 127. 